\title{
Characterization of Heterogeneous Materials with the Confocal Raman AFM*
}

\author{
F. VARGAS AND U. SCHMidT \\ WITec GmbH, Lise-Meitner Str. 6, Ulm 89081, Germany ${ }^{\dagger}$
}

\section{Introduction}

Many technically interesting materials are heterogeneous on a microscopic scale. By combining confocal microscopy with the Raman spectroscopy, the chemical composition of such materials can be analyzed without laborious sample preparation. The additional atomic force microscopy (AFM) techniques added to the confocal Raman microscope allow the real time imaging of the surface topography with resolution below the diffraction limit.

\section{Results}

Figure 1 shows the chemical composition of an adhesive paper label. The image is recorded in the Raman spectral imaging mode by using the depth scan capability of a confocal microscope. In the Raman spectral
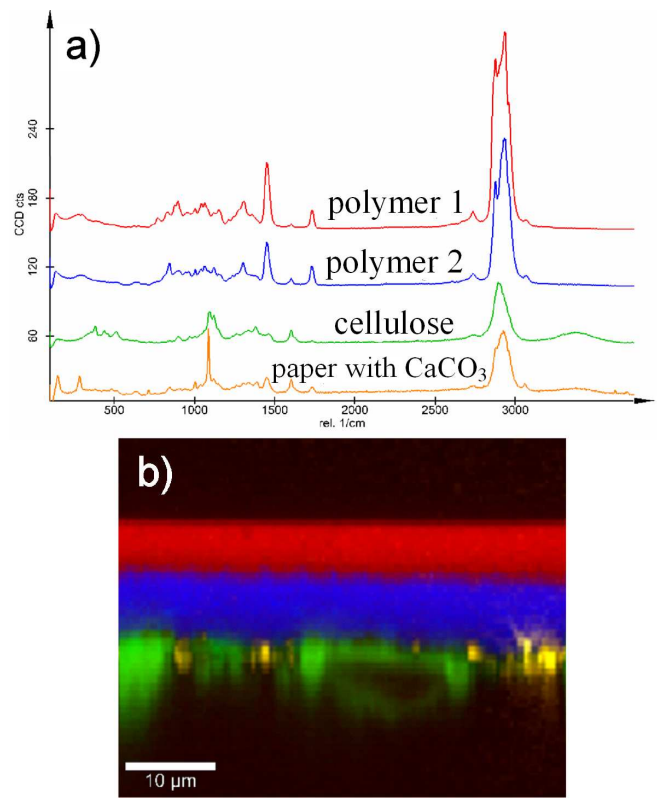

Fig. 1. Adhesive paper label. Raman spectra of the coatings (a) and depth profile of the polymer coatings on the white paper (b).

\footnotetext{
* contact: Agnieszka Kowalczyk, 41-710 Ruda Śląska, Poland, e-mail: kowalczyk@lot-oriel.pl

$\dagger$ www.witec.de
}

imaging a complete Raman spectrum is recorded at every image pixel, resulting in a two-dimensional array of the Raman spectra. By analyzing specific features of the recorded Raman spectra, various images can be calculated from the array of spectra. Two different polymer layers are clearly visible. Due to the confocal principle, the thickness of each polymer layer can be determined. The distinctive separation of the two polymer layers indicates that they are immiscible. The support for these two polymer layers is paper. Paper consists mainly of cellulose (green spectrum). To provide the paper a white surface finish, calcite is added to the paper fabrication process. Traces of calcite are displayed in yellow color.
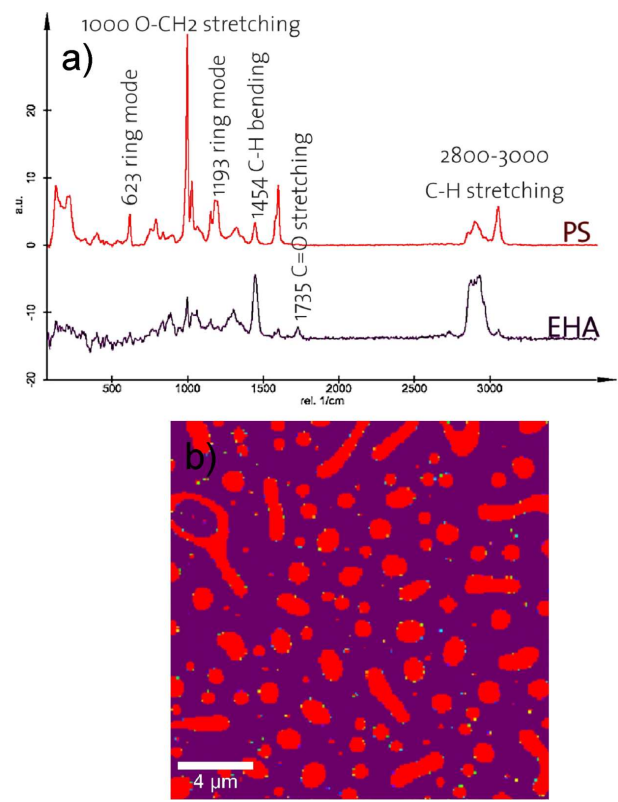

Fig. 2. Confocal Raman imaging of a polymer blend (PS) and acrylate (EHA). Raman spectra of PS and EHA (a) and color coded chemical image of the blend (b).

Beside the nondestructive studies of the bulk composition of materials, it is also possible to analyze the composition of ultrathin films with the confocal Raman microscope. In these studies complete Raman spectra are recorded in one focal plane at every image pixel. Figure 2 a shows the Raman spectra of polystyrene (PS) and 


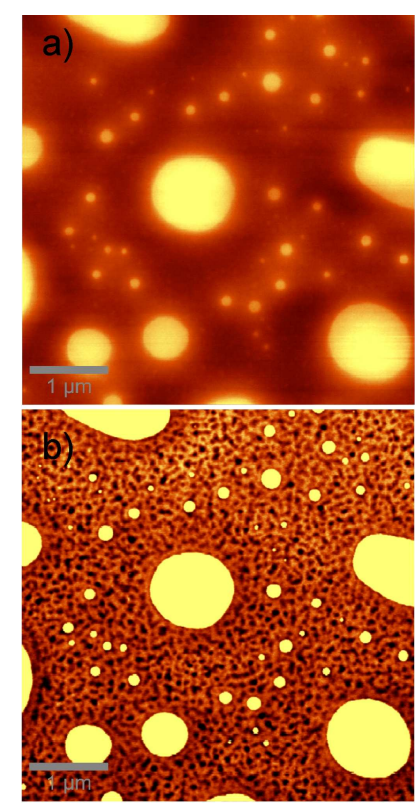

Fig. 3. High resolution AFM images of the thin film of the polymer blend PS-EHA: topography (a) and phase image (b).

acrylate measured on a spin coated thin film of the polymer blend on a glass substrate. The distribution of the two phases within the thin film is shown in Fig. 2b. Each polymer of the blend forms well defined domains in the film. An overlap of the individual components was not detected, indicating that both polymers have formed an interface with the glass substrate while de-wetting. By simply turning the microscope turret, the confocal Raman microscope is transformed into an AFM. The surface topography of the blend is shown in Fig. 3a. The domains of polystyrene are only $70 \mathrm{~nm}$ high and appear as islands surrounded by a $30 \mathrm{~nm}$ high acrylate phase. The simultaneously recorded phase image (shown in Fig. 3b) reveals an additional fine structure within the acrylate phase. By using the combination of three unique measuring techniques in one instrument, a detailed analysis of the chemical composition and topographic fine structure of the film is possible. A film thickness of only $30 \mathrm{~nm}$ was enough to acquire a distinctive Raman spectrum of a polymeric compound with only $50 \mathrm{~ms}$ integration time per spectrum. The film thickness was determined by mechanically scratching the film and subsequent step height measurement with the AFM.

\section{Conclusion}

As demonstrated by means of the examples above, a combination of complementary measuring techniques in one instrument is indispensable in the characterization of heterogeneous materials and their implementation in functional devices. The confocal Raman AFM, which combines the Raman spectroscopy with high-resolution topography imaging, is a powerful tool to analyze complex materials. The surface topography can be imaged at the highest resolution with the AFM and various materials contributing to the surface composition can be chemically identified with the confocal Raman microscope. 